\title{
Evidence for creation of strong electromagnetic fields in relativistic heavy-ion collisions ${ }^{\star}$
}

\author{
V. Toneev ${ }^{\mathrm{a}}$, O. Rogachevsky, and V. Voronyuk \\ Joint Institute for Nuclear Research, Dubna, Russia
}

Received: 7 September 2015 / Revised: 21 April 2016

Published online: 24 August 2016

(c) The Author(s) 2016. This article is published with open access at Springerlink.com

Communicated by D. Blaschke

\begin{abstract}
It is proposed to identify the strong electric field created during relativistic collisions of asymmetric nuclei via observation of pseudorapidity and transverse-momentum distributions of hadrons with the same mass but opposite charges. The detailed calculation results for the directed flow within the Parton-Hadron String Dynamics model are given for $\mathrm{Cu}-\mathrm{Au}$ interactions at the NICA collision energies of $\sqrt{s_{N N}}=9$ and $5 \mathrm{GeV}$. The separation effect is observable at $9 \mathrm{GeV}$ as clearly as at $200 \mathrm{GeV}$.
\end{abstract}

It was demonstrated in ref. [1] that charged particles created in relativistic heavy-ion collisions may produce extremely strong electromagnetic field strength. In particular, in peripheral $\mathrm{Au}-\mathrm{Au}$ collisions at the energy $\sqrt{s_{N N}}=200 \mathrm{GeV}$ in the very initial interaction state this strength reaches $\left|e B_{y}\right| / m_{\pi}^{2} \sim 5=5 \cdot 10^{18}$ Gauss, which exceeds every value reachable in the earth conditions and even may be higher than the fields created in magnetars. However, the subsequent analysis of $\mathrm{Au}+\mathrm{Au}$ collisions in the range up to the top RHIC energy has observed no visible effect of the strong electromagnetic interactions on global characteristics, in particular, for such a sensitive quantity as the elliptic flow. The reason of that is not a very short interaction time of the system, as could be naively expected, but rather a compensation effect between electric and magnetic components of acting electromagnetic forces, as found in ref. [2]. Thus, the question of wether so strong electromagnetic fields are really created in high-energy nuclear collisions remains open.

Recently, it has been found that such compensation is absent in asymmetric relativistic collisions due to the difference in the number of protons in colliding nuclei $[3,4]$. In particular, in $\mathrm{Cu}+\mathrm{Au}(200 \mathrm{GeV})$ collisions the directed flow (the first flow harmonic $v_{1}\left(\eta, p_{t}\right)$ ) exhibits an electric charged particle dependence. As follows from the results presented in fig. 1, the electric field in the central region of the overlapping area has a specific tendency to go from $\mathrm{Au}$ to $\mathrm{Cu}$ and this effect can be observed at the nominal NICA energy $\sqrt{s_{N N}}=9 \mathrm{GeV}$, too. Indeed, the

\footnotetext{
* Contribution to the Topical Issue "Exploring strongly interacting matter at high densities - NICA White Paper" edited by David Blaschke et al.

a e-mail: toneev@theor.jinr.ru
}

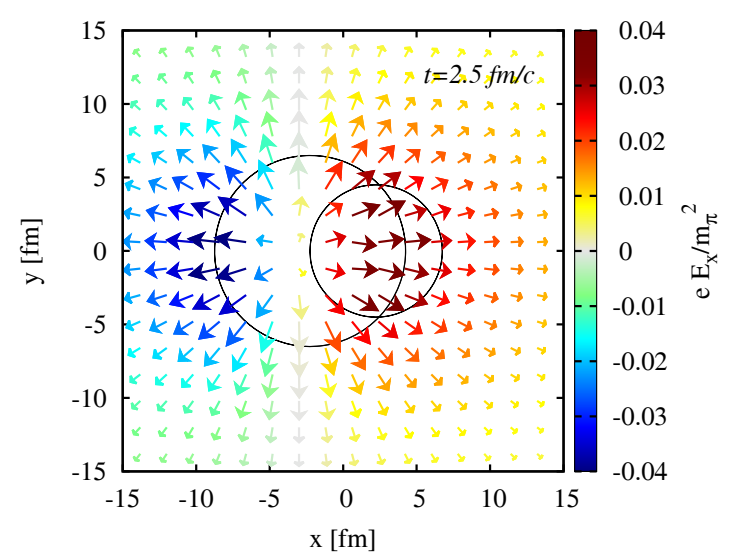

Fig. 1. Electric field generated in the transverse plane of $\mathrm{Cu}+\mathrm{Au}$ collisions at $\sqrt{s_{N N}}=9 \mathrm{GeV}, b=4.5 \mathrm{fm}$ and $t=$ $2.5 \mathrm{fm} / c$. The direction of the arrows indicates the field direction projected onto the reaction plane and the length is proportional to the electromagnetic strength shown in color.

strength of the induced electric field is strongly asymmetric within the region of overlapping nuclei, which may result in observable asymmetry of hadrons with opposite charges.

As noted in [2], the electromagnetic field (EMF) is formed predominantly by spectators in the early time during the mutual passage of the two colliding nuclei. Since the number of spectators increases with the impact parameter $b$, the magnetic field should also increase in more peripheral collisions and decrease gradually with $b$. In addition, the directed flow $v_{1}$ is getting larger when one proceeds to more peripheral collisions. Thus, this experiment is more promising at large impact parameters. 


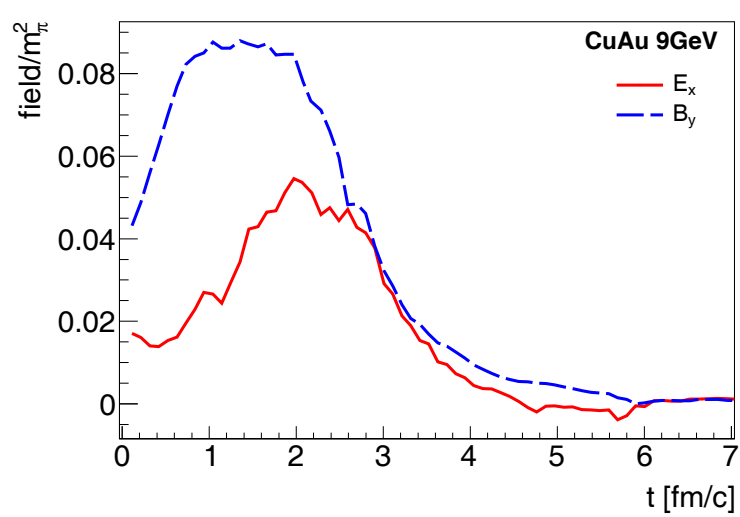

Fig. 2. The time dependent components of the average electric $E_{x}$ and magnetic $B_{y}$ field generated in the center of $\mathrm{Cu}+\mathrm{Au}$ collision at $b=4.5 \mathrm{fm}$ and $\sqrt{s_{N N}}=9 \mathrm{GeV}$.
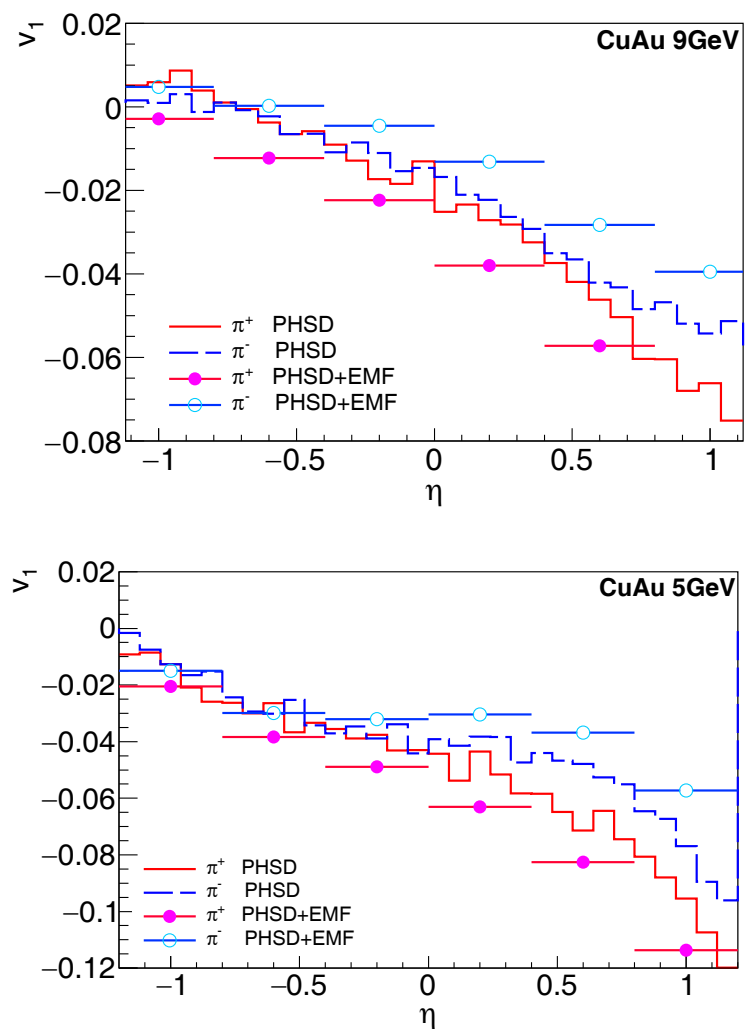

Fig. 3. Pseudorapidity distribution of positive and negative pions created in $\mathrm{Cu}+\mathrm{Au}$ collisions at $\sqrt{s_{N N}}=9$ (top figure) and 5 (bottom) $\mathrm{GeV}$ and the impact parameter interval 4.4-9.5 fm. Histograms are the result of the standard PHSD transport approach, points are the results where the electromagnetic force is additionally included.

As is seen from fig. 2, the average strength of the dominant components of the electric $\left\langle E_{x}\right\rangle$ and magnetic $\left\langle B_{y}\right\rangle$ fields reaches in the time interval $1-2 \mathrm{fm} / c$ maximal values $\left\langle e E_{x}\right\rangle \approx 0.050 m_{\pi}^{2} \mathrm{GeV}^{2}$ and $\left\langle e B_{y}\right\rangle \approx 0.085 m_{\pi}^{2} \mathrm{GeV}^{2}$. Other components are practically negligible. These values are obtained in the spatial cylinder with radius $R=2 \mathrm{fm}$ and length $|z|<2.5 / \gamma$ fm passing through the center of colliding nuclei. The maximal average energy density reached in the same cylinder is about 1.6 and $0.9 \mathrm{GeV} / \mathrm{fm}^{3}$
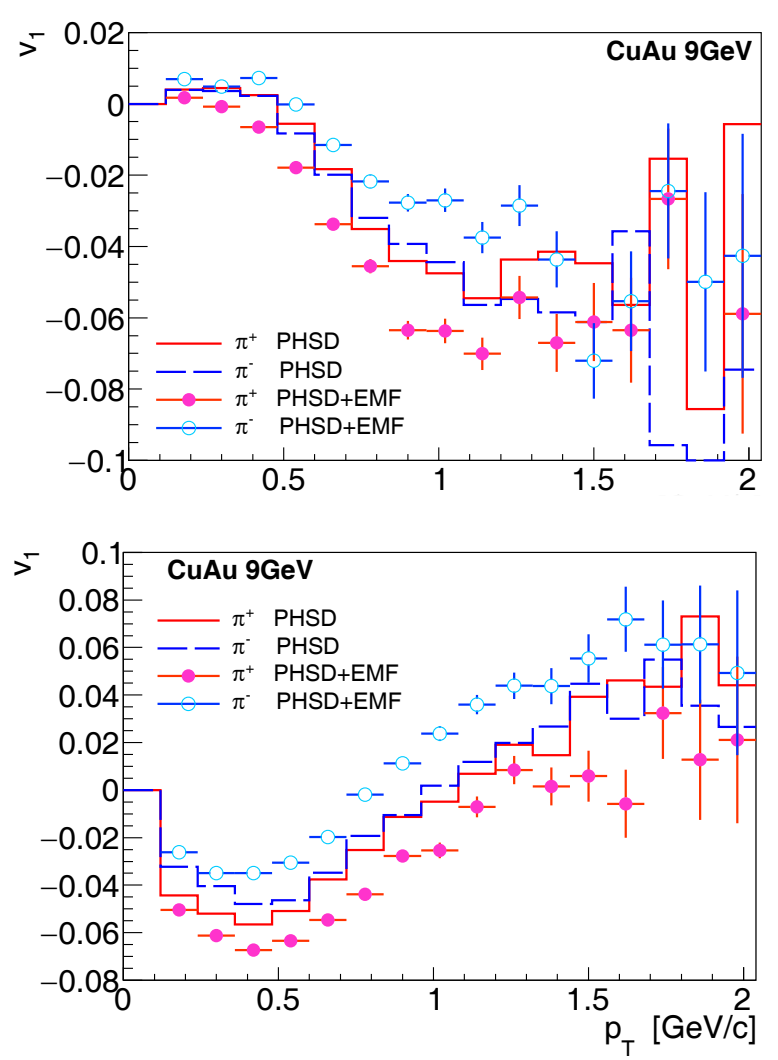

Fig. 4. Charge-dependent $p_{t}$ distributions of pions from asymmetric $\mathrm{Cu}+\mathrm{Au}$ collisions at $\sqrt{s_{N N}}=9 \mathrm{GeV}$ for backward (upper panel) and forward (bottom panel) emission. The parameters used and notation are as in fig. 3.

for collision energy 9 and $5 \mathrm{GeV}$, respectively, that justifies the use of the partonic version of the string dynamic model.

In this respect, on the basis of the Parton Hadron String Dynamics model [5-7] we calculated various characteristics of asymmetric $\mathrm{Cu}+\mathrm{Au}$ collisions at the NICA energy $\sqrt{s_{N N}}=9$ and $5 \mathrm{GeV}$. As is seen from fig. 3, without accounting for the created EMF the $\eta$ distributions for $\pi^{+}$and $\pi^{-}$are very close to each other (the difference is coming only from different mean multiplicity of these pions), but the inclusion of the electromagnetic field results in a sizable separation of these distributions. Note that the detector acceptance is taken into account here. At lower energy, $5 \mathrm{GeV}$, the separation effect is weaker: the difference between $v_{1}(\eta)$ distributions calculated with and without the EMF is within statistical error bars.

The transverse-momentum distributions of the directed flow $v_{1}$ for pions created at $\sqrt{s_{N N}}=9 \mathrm{GeV}$ are presented in fig. 4. It is remarkable that $v_{1}\left(p_{t}\right)$ differs in the backward $(\eta<0)$ and forward $(\eta>0)$ directions. For $\eta<0$ (the Au side) the low-momentum $\left(p_{t}<1 \mathrm{GeV} / c\right)$ pions are dominant while in the opposite direction the $p_{t}$ distribution is an increasing function with some minima at $p_{t} \sim 0.5 \mathrm{GeV} / c$. In both cases the charge separation is well visible in fig. 4 and it is getting larger for higher values of $p_{t}$. 

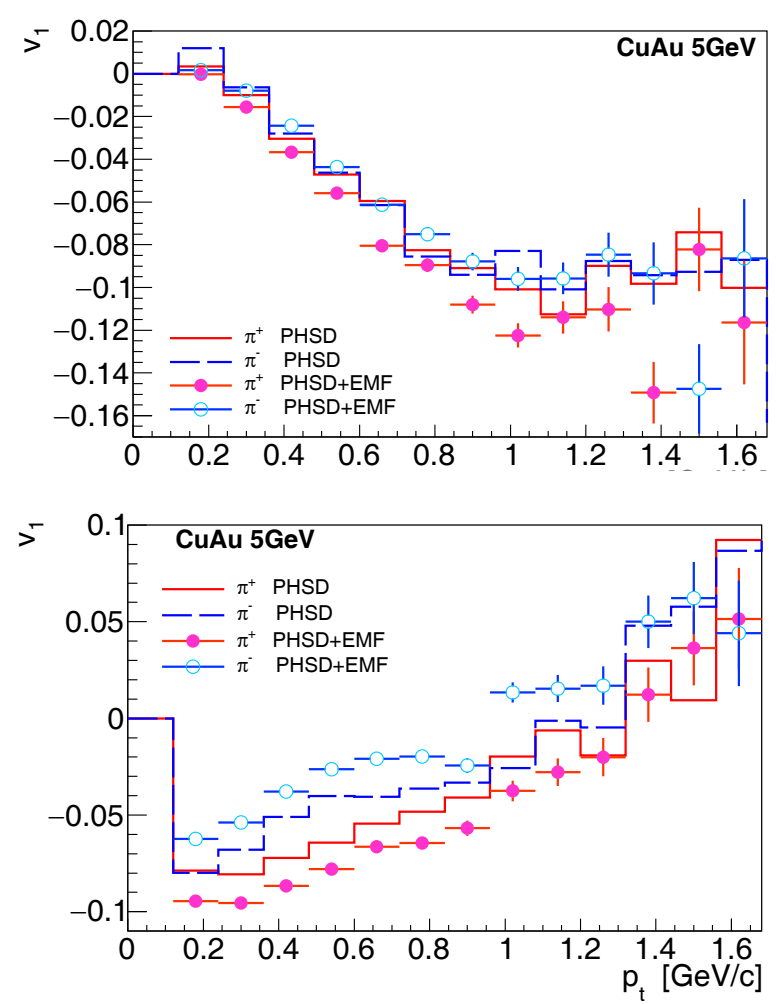

Fig. 5. Charge-dependent $p_{t}$ distributions of pions from asymmetric $\mathrm{Cu}+\mathrm{Au}$ collisions at $\sqrt{s_{N N}}=5 \mathrm{GeV}$ for backward (upper panel) and forward (bottom panel) emission. Notation as in fig. 3.

The pion transverse-momentum distributions for the collision energy $5 \mathrm{GeV}$ are shown in fig. 5 . It is clearly seen that though the functional behavior of $v_{1}\left(p_{t}\right)$ is quite similar to that in fig. 4 , the magnitude of the pion directed flow $v\left(p_{t}\right)$ is suppressed at lower energy resulting in smaller separation effect. It is noteworthy that the magnitude of $v_{1}$ is a weakly increasing function above $\sqrt{s_{N N}}=9 \mathrm{GeV}$ and practically coincides with appropriate values at the collision energy $200 \mathrm{GeV}$ [3].

Similar effects are observed for $K^{+}$and $K^{-}$mesons. However, for a proton-antiproton pair this is not the case since the strong interaction of $p$ and $\bar{p}$ is quite different.
The particularity of the NICA energy range is that the particle creation occurs at a high baryon density or a large baryonic chemical potential. In addition, the electric charge chemical potential is also quite important since we are interested in hadrons with opposite electric charges. Future study of these effects, especially in the partonic phase, is of great interest.

Thus, we propose to measure the pseudorapdity $v_{1}(\eta)$ and transverse-momentum $v_{1}\left(p_{T}\right)$ distributions of the directed flow for identified pairs of hadrons (at least for $\pi^{+}$, $\pi^{-}$and $K^{+}, K^{-}$mesons) in the NICA energy range, which could first evidence a new physical effect - the formation of extremely strong electromagnetic fields in relativistic heavy-ion collisions. The top NICA energy is favorable for such measurements. The realization of this experiment implies that two ions of different kinds may interact in the collider creating a very high electromagnetic field. As an estimate shows, the planned luminosity $L=10^{27} \mathrm{~cm}^{-2} \mathrm{~s}^{-1}$ resulting in the collision rate $(\sigma L \epsilon)=500$ event/s makes this experiment feasible at the NICA collider.

Open Access This is an open access article distributed under the terms of the Creative Commons Attribution License (http://creativecommons.org/licenses/by/4.0), which permits unrestricted use, distribution, and reproduction in any medium, provided the original work is properly cited.

\section{References}

1. V. Skokov, A. Illarionov, V. Toneev, Int. J. Mod. Phys. A 24, 5925 (2009).

2. V. Voronyuk, V.D. Toneev, W. Cassing, E.L. Bratkovskaya, V.P. Konchakovski, S.A. Voloshin, Phys. Rev. C 83, 054911 (2011).

3. V. Voronyuk, V.D. Toneev, S.A. Voloshin, W. Cassing, Phys. Rev. C 90, 064903 (2014).

4. Y. Hirono, M. Hongo, T. Hirano, Phys. Rev. C 90, 021903(R) (2014).

5. W. Cassing, E. Bratkovskaya, Phys. Rev. C 78, 034919 (2008).

6. W. Cassing, E. Bratkovskaya, Nucl. Phys. A 831, 215 (2009).

7. W. Cassing, E. Bratkovskaya, Nucl. Phys. A 856, 162 (2011). 